\title{
Microbiological Factors in Assessment of Health Risk Evolution Caused by Various Factors
}

\author{
Nina Zaitseva, Pavel Shur*, Dmitriy Kiryanov, Marat Kamaltdinov and Vladimir Chigvintsev \\ Federal Scientific Center for Medical and Preventive Health Risk Management Technologies, Russia
}

Submission: August 21, 2017; Published: August 23, 2017

*Corresponding author: Pavel Shur, academic secretary, Federal Scientific Center for Medical and Preventive Health Risk Management Technologies, Russian Federation, 82 Monastyrskaya St, Perm, Russia, Tel +7(342)238-33-37; Fax: +7(342)237-25-34; Email: shur@fcrisk.ru

Keywords: Health risk assessment; Microbiological factors; Risk evolution

\section{Introduction}

Methodical approaches to predicting health risks which apply evolution models allow to assess health risk caused by various environmental factors including microbiological for differentiated periods of exposure to them [1-4]. Modeling of health risk evolution is based on creation of models which describe accumulation of damage done by negative impacts exerted by environmental factors. Such modeling is performed via a technique in which damage parameter is given in a form of conventional differential equations for health damage evolution [5]. Contemporary development of risk assessment and calculation theory is determined by considering risks as a dynamic process which reflects accumulation of probable damage to the body organs and systems caused by various environmental factors influence.

Health damage risk is given as a combination of occurrence probability and gravity:

$$
R_{j}=P_{j} \cdot g_{j}, \text { where }
$$

$R_{j}$ is health risk caused by $\mathrm{j}$-th non-infectious disease;

$P_{j}$ is j-th non-infectious disease probability;

$g_{j}$ is $\mathrm{j}$-th non-infectious disease gravity.

Evolution modeling made it possible to formalize predicting procedure for non-carcinogenic risk caused by chemical environmental factors and to give the evolution model as follows:

$$
d R_{j}=\alpha_{j} R_{j}+\sum_{i} \beta_{i j} F_{i j}\left(x_{i}\right) \text { where }
$$

$R_{j}$ is risk of functional disorders in $\mathrm{j}$-th organ or system;

$\alpha_{j}$ is a coefficient which allows for evolution of functional disorders risk in $\mathrm{j}$-th organ or system due to natural causes;

$\beta_{i j}$ is a coefficient showing gravity of influence exerted by $i$-th chemical factor on evolution of functional disorders risks in $\mathrm{j}$-th organ or system due to natural causes;

$F_{i j}\left(x_{i}\right)$ is a function of influence exerted by i-th chemical factor on evolution of functional disorders risks in j-th organ or system;

$x_{i}$ is i-th chemical factor exposure.

This fundamental model can be relevant for a number of other factors, for example, physical ones (noise and electromagnetic radiation) and lifestyle factors (smoking or alcohol abuse). However, modeling of risk caused by microbiological factors which lead to infectious diseases requires allowing for their certain peculiarities. Such peculiarities are as follows:

A. Microbiological factors cause infectious diseases while chemical ones can only influence their occurrence as they induce functional disorders in the body, including protective barriers disorders; 
B. Infectious diseases risk results from exposure to microbiological factors when the body barrier functions are damaged;

C. Negative effects caused by exposure to other factors are accumulated over time, and negative effects caused by most microbiological factors are generally not accumulated as functions recover actively after an infection is over.

Microbiological risks assessment has another distinctive feature as exposure in it is given as a probability that microorganisms can enter a recipient's body in a dose which is higher than a minimal infective one. Up to now, microbiological hazard factors have not been included into health risk evolution modeling. But if interactions between microbiological and other hazard factors were taken into account in health risk prediction with the help of evolution models, it could make for creation of exposure scenarios which were closer to real life conditions and for more comprehensive health risk assessment. Fundamental methodical approaches stated in the paper are aimed at solving this task.

\section{Fundamental Methodical Approaches to Prediction} of Risk caused by Microbiological and Other Hazard Factors Influence

Generally, probability of non-infectious diseases occurrence is a dynamic process; its rate depends on negative effects related to hazard factors influence and consequences of infectious diseases occurrence:

$$
\frac{d P_{j}}{d t}=f\left(P_{j}, x_{i}, P_{k}^{I n f}\right), \text { where }
$$

$P_{j}$ is $\mathrm{j}$-th non-infectious disease probability;

$x_{i}$ is exposure to i-th other factor;

$P_{k}^{\operatorname{Inf}}$ is k-th infectious diseases probability.

Or it is given as evolution equation:

$$
\frac{d P_{j}}{d t}=\alpha_{j} P_{j}+\sum_{i} \beta_{i j} f_{i j}\left(x_{i}\right)+\sum_{k} \gamma_{k j} \phi_{k j}\left(P_{k}^{I n f}, d_{k}\right), \text { where }
$$

$P_{j}$ is j-th non-infectious disease probability;

$\alpha_{j}$ is a coefficient allowing for evolution of $\mathrm{j}$-th noninfectious disease probability due to natural causes;

$\beta_{i j}$ is a coefficient for influence exerted by $\mathrm{i}$-th other factor on evolution of $\mathrm{j}$-th non-infectious disease probability;

$f_{i j}\left(x_{i}\right)$ is a function of influence exerted by i-th other factor on evolution of $\mathrm{j}$-th non-infectious disease probability;

$x_{i}$ is i-th other factor exposure; $\gamma_{l j}$ is a coefficient for influence exerted by k-th infectious disease on evolution of $\mathrm{j}$-th non-infectious disease probability;

$\phi_{k j}\left(P_{k}^{\operatorname{Inf}}, d_{k}\right)$ is a function of influence exerted by k-th infectious disease evolution of $\mathrm{j}$-th non-infectious disease probability;

$P_{k}^{I n f}$ is k-th infectious disease probability;

$d_{k}$ is k-th microbiological factor exposure.

Infectious Disease Probability is Determined by Exposure To A Relevant Microbiological Factor and the State of the Body's Barrier Functions

$$
P_{k}^{I n f}=\frac{p\left(d_{k}>d_{k 0}\right)}{(1-D)} \text {, where }
$$

$p\left(d_{k}>d_{k 0}\right)$ is probability of k-th microbiological factor introduction in a dose which is higher than a minimal infective one;

$d_{k}$ is k-th microbiological factor exposure.

$d_{k 0}$ is a threshold value for $\mathrm{k}$-th microbiological factor exposure.

$D$ is the state of the body's barrier functions.

Disorders in barrier functions are given by the following ratio:

$$
\frac{\not D}{d}=\alpha_{D} D+\sum_{i} \beta_{D} f_{D}\left(x_{i}\right), \text { where }
$$

$D$ is the state of the body's barrier functions;

$\alpha_{D}$ is a coefficient allowing for evolution of the body barrier function state due to natural causes;

$\beta_{i D}$ is a coefficient for influence exerted by i-th other factor on evolution of the body barrier function state;

$f_{i D}\left(x_{i}\right)$ is a function of influence exerted by i-th chemical factor on evolution of the body barrier function state.

The given ratios make it possible to formalize additional risk calculation ( )in a form of models considering the probability of negative responses taking into account their severity under combined effects exerted by microbiological factors and other hazard factors. The risk characteristic includes the calculation of additional health risk values associated with the influence of the set of studied factors. The additional risk of health disorders associated with adverse effects of the individual environmental factors can be determined according to the formula:, 


$$
\Delta R_{j t}=R_{t}-R_{t}^{b}
$$

where $R_{t}^{b}$ - additional risk of the health disorder $\mathrm{j}$ in the moment of time t.

To Evaluate the Risk Level, the Following Reduced Health Index is Calculated, That is Connected with the Factors Effects

$$
\widetilde{R}_{t}=\frac{\Delta R_{t}}{1-R_{t}^{b}}
$$

The given risk index characterizes the probability of health disorders under the environmental impact and taking into account the growth of the general health risk with the increase of exposure time.

\section{Prospects and Conditions of Health Risk Prediction Allowing for Microbiological Hazard Factors in Evolution Models}

The discussed approaches can be treated as the first attempt to give grounds for introduction of microbiological hazard factors into health risk assessment with the help of evolution models. Formulation of a hypothesis on participation which microbiological factors have in health risk occurrence and creation of fundamental approaches to assessment of health risk caused by microbiological factors allows to identify basic research trends which are necessary conditions for the development of these assumptions.

Nowadays the suggested approaches are greatly uncertain and if we want to decrease this uncertainty we need to clarify the nature and volume of functional disorders in organs and systems caused by infectious diseases; to detect quantitative parameters of evolution in the state of the body barrier function due to natural causes; to parameterize infectious diseases gravity dependence on a microbe agents dose for recipients with different body barrier functions state; to assess influence exerted by chemical, physical, and other factors on evolution of the body barrier function state.

\section{Conflict of interest}

The authors declare that they have no conflict of interest.

\section{References}

1. Zaitseva NV, Trusov PV, Shur PZ, Kiryanov DA, Chigvintsev VM, et al. (2013) Methodical approaches to assessment of health risk caused by influence exerted by various environmental factors on population health basing on evolution models. Health risk analysis 1: 3-11.

2. Methodical guidelines 2.2.10.0059-12. Assessing population health risks caused by transport noise.

3. Methodical guidelines 2.1.10.0061-12. Assessing population health risks caused by variable electromagnetic fields (up to $300 \mathrm{GHz}$ ) in settlements.

4. Methodical guidelines 2.1.10.0062-12. Quantitative assessment of non-carcinogenic risk under exposure to chemicals on the basis of evolution models creation.

5. Trusov PV, Zaitseva NV, Kiryanov DA, Kamaltdinov MR, Zinker M Yu, et al. (2012) A mathematical model for evolution of human functional disorders influenced by environment factors. Mathematical biology and bioinformatics 7(2): 589-610.

\section{Your next submission with Juniper Publishers will reach you the below assets}

- Quality Editorial service

- Swift Peer Review

- Reprints availability

- E-prints Service

- Manuscript Podcast for convenient understanding

- Global attainment for your research

- Manuscript accessibility in different formats

( Pdf, E-pub, Full Text, Audio)

- Unceasing customer service

Track the below URL for one-step submission https://juniperpublishers.com/online-submission.php 